\title{
Spectral Properties of Certain Composition Operators Arising in Statistical Mechanics
}

\author{
D. H. Mayer
}

Institut für Theoretische Physik, RWTH Aachen, D-5100 Aachen, Federal Republic of Germany

\begin{abstract}
By applying the theory of linear positive operators in a Banach space we derive spectral properties of certain composition operators in the Banach space $A_{\infty}(\Omega)$ of holomorphic functions over some domain $\Omega \subset \mathbb{C}^{n}$. Examples of such operators are provided by the so called generalized transfer matrices of classical one-dimensional lattice systems.
\end{abstract}

\section{Introduction}

It was shown in a series of papers [1-5] how one can define generalized transfer matrices for one-dimensional classical lattice systems even if the interaction between spins on different lattice sites is not of finite range. These transfer matrices are given as abstract linear trace class operators acting in Banach spaces of holomorphic functions of finite or infinite many complex variables. The general form of such a transfer matrix $T$ is given as follows

$$
T f(z)=\sum_{k=1}^{m} \varphi_{k}(z) f \circ \psi_{k}(z) .
$$

In (1) the functions $\varphi_{k}$ and $f$ are holomorphic in the variable $z \in \Omega$, where $\Omega$ is some open bounded region in $\mathbb{C}^{n}$ or in some complex Banach space $B$. The $\psi_{k}$ 's are holomorphic mappings of $\Omega$ into itself, such that $T$ defines a linear operator in the Banach space $A_{\infty}(\Omega)$ of holomorphic functions over $\Omega$. It was shown in [5] that under certain conditions on the mappings $\psi_{k}$ the operator $T$ as defined in (1) defines a nuclear operator of order zero which insures that $T$ and all powers $T^{n}$, $n \geqq 1$, have a trace.

In statistical mechanics one knows that the physical properties of a system which allows for a transfer matrix $T$ are determined by the spectral properties of this operator [6]. For the one-dimensional systems for which a transfer matrix as in (1) exists one knows from different methods that all thermodynamic potentials are analytic functions in all parameters of interest, like for instance the temperature. This reflects the fact that such a system does not have a phase transition. 
From this one expects that the highest eigenvalue of $T$ is in fact analytic in such parameters.

Now it is amusing to see how one can derive such spectral properties directly for operators of the kind defined in (1). It should be clear that there is some mathematical reason why this class of operators shows such a spectral behaviour. This is the problem we want to discuss in this paper. We will show that there is a wide class of what we shall call composition operators which show all the same spectral behaviour.

The method which we shall apply is the theory of positive operators in a Banach space which leave a cone invariant. This is a generalization of the well known results of Perron-Frobenius [7] on positive and strictly positive matrices in finite dimensional spaces and of Jentzsch [8] on integral operators with positive kernel. The theory as we use it here was mainly developped by Krein and Rutman [9] as well as by Krasnoselskii and Ladyzenskii [10]. The last mentioned authors have studied the so called $u_{0}$-positive operators which have properties similar to those of strictly positive matrices in finite dimensional spaces.

The main part of this paper will in fact be to show that operators $T$ as in (1) give rise to such $u_{0}$-positive operators.

For the readers convenience we will repeat in a first section very briefly the main definitions and results on positive and $u_{0}$-positive operators in a Banach space $B$.

In a second section we will then show how our composition operators $T$ in (1) fit into this scheme. We will discuss under what conditions on the $\varphi_{k}$ and the $\psi_{k}$ the operator $T$ defines an $u_{0}$-positive operator. For these operators then we get immediately the spectral properties we expected.

We will restrict ourselves in this paper to the case where $\Omega$ is an open, bounded, simply connected region in $\mathbb{C}^{n}$ with $n<\infty$. The case $\Omega \subset B$ with $B$ some complex Banach space can be treated in a similar way if one changes the arguments in the appropriate way as we did already in [5].

\section{Positive and $u_{0}$-Positive Operators}

Let $B$ be a Banach space, complex or real, and let $K$ be a proper cone in $B$. (For more details see for instance [11] or [12].) The cone $K$ is called reproducing if $B=K-K$. The cone $K$ defines a partial ordering $\leqq$ in $B: x \leqq y$ iff $y-x \in K$. A linear operator $T: B \rightarrow B$ is called a positive operator iff $T K \subset K$, that means $T$ leaves the cone invariant. Let $u_{0} \in K, u_{0} \neq 0$. The positive operator $T$ is called $u_{0}$-upper bounded if there exist for every $x \in K, x \neq 0$ a natural number $p \geqq 1$ and a strictly positive number $\alpha>0$ such that

$$
T^{p} x \leqq \alpha u_{0}
$$

In a similar way $T$ is called $u_{0}$-lower bounded if there exist to every $x \in K, x \neq 0$ a natural number $q \geqq 1$ and a strictly positive number $\beta>0$ such that

$$
T^{q} x \geqq \beta u_{0}
$$

A very interesting class of positive operators were introduced in [10]. They are the $u_{0}$-positive operators and are defined as follows: 
Let $u_{0} \in K, u_{0} \neq 0$. A positive operator $T$ is called $u_{0}$-positive if there exist for every $x \in K, x \neq 0$ a natural number $p \geqq 1$ and positive numbers $\alpha, \beta>0$ such that

$$
\beta u_{0} \leqq T^{p} x \leqq \alpha u_{0} .
$$

One can show [13] that every positive operator which is $u_{0}$-upper and lower bounded is $u_{0}$-positive. For these operators the Perron-Frobenius and Jentzsch Theorems have a natural generalization:

Theorem A. Let $K$ be a reproducing cone in the real Banach space $B$. Let $T: B \rightarrow B$ be a $u_{0}$-positive compact operator in $B$. Choose $p$ and $\beta>0$ such that $T^{p} u_{0} \geqq \beta u_{0}$. Then

a) there exists exactly one eigenvector $x_{0}$ of $T$ in $K, T x_{0}=\lambda_{0} x_{0}$ with $\lambda_{0}$ strictly positive and $\lambda_{0} \geqq \sqrt[p]{\beta}$.

b) $\lambda_{0}$ is a simple eigenvalue and all other eigenvalues of $T$ are in absolute value strictly smaller than $\lambda_{0}$.

The proof of this theorem can be found in the book of Krasnoselskii [14].

\section{The Spectrum of Some Composition Operators}

Let $\Omega$ be some open, bounded, simply connected domain in $\mathbb{C}^{n}$. We denote by $A_{\infty}(\Omega)$ the Banach space of holomorphic functions on $\Omega$ which are continuous on $\bar{\Omega}$ together with the sup norm. Let $z_{0} \in \Omega$. Then we denote by $\Omega_{\mathbb{R}}\left(z_{0}\right)$ the set

$$
\Omega_{\mathbb{R}}\left(z_{0}\right):=\left(z_{0}+\mathbb{R}^{n}\right) \cap \Omega .
$$

We remark that $\Omega_{\mathbb{R}}\left(z_{0}\right)$ is a set of uniqueness for every $f \in A_{\infty}(\Omega)$ [15]. Furthermore we define the following real subspace $A_{\infty}\left(\Omega_{\mathbb{R}}\left(z_{0}\right)\right)$ of the space $A_{\infty}(\Omega)$ :

$$
A_{\infty}\left(\Omega_{\mathbb{R}}\left(z_{0}\right)\right):=\left\{f \in A_{\infty}(\Omega): f(z) \in \mathbb{R} \forall z \in \Omega_{\mathbb{R}}\left(z_{0}\right)\right\} .
$$

Clearly $A_{\infty}\left(\Omega_{\mathbb{R}}\left(z_{0}\right)\right)$ is a real Banach space with the induced sup norm.

We define next an open set $A_{\infty}^{+}\left(\Omega_{\mathbb{R}}\left(z_{0}\right)\right)$ in $A_{\infty}\left(\Omega_{\mathbb{R}}\left(z_{0}\right)\right)$ :

$$
A_{\infty}^{+}\left(\Omega_{\mathbb{R}}\left(z_{0}\right)\right):=\left\{f \in A_{\infty}\left(\Omega_{\mathbb{R}}\left(z_{0}\right)\right): f(z)>0 \forall z \in \Omega_{\mathbb{R}}\left(z_{0}\right)\right\} .
$$

Finally we denote by $H_{i n}(\Omega)$ the set of all holomorphic mappings $\psi: \Omega_{1} \rightarrow \Omega$ where $\Omega_{1}$ is some open neighbourhood of the closure $\bar{\Omega}$ of $\Omega$.

Let $\psi \in H_{\text {in }}(\Omega)$. Denote by $z^{*}$ the unique fixed point of $\psi[16]$. Let $\Omega_{\mathbb{R}}\left(z^{*}\right)$ be defined as in (5). In what follows we are mostly interested in the following subset $H_{i n}^{\mathbb{R}}(\Omega)$ of the set $H_{i n}(\Omega)$ :

$$
H_{\text {in }}^{\mathbb{R}}(\Omega):=\left\{\psi \in H_{\text {in }}(\Omega): \overline{\psi\left(\Omega_{\mathbb{R}}\left(z^{*}\right)\right)} \subset \Omega_{\mathbb{R}}\left(z^{*}\right)\right\} .
$$

Let $\psi_{k}, k=1, \ldots, m$ be elements in the set $H_{i n}^{\mathbb{R}}(\Omega)$ such that there exists a $k_{0}$ with the property that for all $k=1, \ldots, m$ we have $z_{k}^{*} \in \Omega_{\mathbb{R}}\left(z_{k_{0}}^{*}\right)$. We then denote this set simply by $\Omega_{\mathbb{R}}$.

Let $\boldsymbol{\alpha}=\left(\alpha_{1}, \alpha_{2}, \ldots\right), \alpha_{i} \in \mathbb{N}$, be a multiindex such that $|\boldsymbol{\alpha}|=\sum_{k=1}^{\infty} \alpha_{k}<\infty$. We denote 
then by $\psi^{\alpha_{1}, \ldots, \alpha_{m}}$ the following holomorphic mapping of $\Omega$ into $\Omega$ :

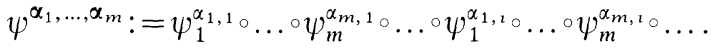

Under the above assumptions it is clear that $\psi^{\boldsymbol{\alpha}_{1}, \ldots, \boldsymbol{\alpha}_{m}}$ is an element of the space $H_{i n}^{\mathbb{R}}(\Omega)$.

Let $f \in A_{\infty}(\Omega)$. Let $H$ be some region with $\bar{H} \subset \Omega$. Let $G_{1}, \ldots, G_{r}$ be the $(2 n-2)$ dimensional analytic nullsets of $f$ in $\bar{H}$ [17]. We call the mappings $\psi_{1}, \ldots, \psi_{m} \in H_{i n}^{\mathbb{R}}(\Omega)$, such that $z_{k}^{*} \in \Omega_{\mathbb{R}}$ for all $k$, separating if the following is true:

There exists for every $H$ a natural number $N_{0}<\infty$ such that for $N \geqq N_{0}$ and every $z \in \bar{H} \cap \Omega_{\mathbb{R}}$ with $\psi^{\boldsymbol{\alpha}_{1}, \ldots, \boldsymbol{\alpha}_{m}}(z) \in \bigcup_{i} G_{i} \cap \Omega_{\mathbb{R}}$ for some $\boldsymbol{\alpha}_{1}, \ldots, \boldsymbol{\alpha}_{m}$ with $\sum_{i=1}^{m}\left|\boldsymbol{\alpha}_{i}\right|=N$, there exists another mapping $\psi^{\boldsymbol{\beta}_{1} \ldots . \boldsymbol{\beta}_{m}}, \sum_{i=1}^{m}\left|\boldsymbol{\beta}_{i}\right|=N$, such that $\psi^{\boldsymbol{\beta}_{1} \ldots, \boldsymbol{\beta}_{m}}(z) \notin G_{i} \cap \Omega_{\mathbb{R}}$ for
all $1 \leqq i \leqq r$.

After all these definitions we can now formulate our main theorem.

Theorem B. Let $\Omega \subset \mathbb{C}^{n}$ be an open, bounded, simply connected domain. Let $\psi_{1}, \ldots, \psi_{m} \in H_{i n}^{\mathbb{R}}(\Omega)$ such that $z_{k}^{*} \in \Omega_{\mathbb{R}}$ for all $k=1, \ldots, m$. Let $\varphi_{k} \in A_{\infty}^{+}\left(\Omega_{\mathbb{R}}\right) \cap A_{\infty}\left(\Omega_{1}\right)$ with $\bar{\Omega} \subset \Omega_{1}$. Define the linear operator $T: A_{\infty}(\Omega) \rightarrow A_{\infty}(\Omega)$ by

$$
(T f)(z):=\sum_{i=1}^{m} \varphi_{i}(z) f \circ \psi_{i}(z) .
$$

Assume that the mappings $\psi_{k}$ are separating in the sense explained above. Then the highest eigenvalue $\lambda_{0}$ of $T$ is strictly positive and simple. We have $\lambda_{0} \geqq \min _{z \in \bar{\Omega}_{\mathbb{R}}} \sum_{k=1}^{m} \varphi_{k}(z)$. All other eigenvalues of $T$ are in absolute value strictly smaller than $\lambda_{0}$.

We are going to prove Theorem B in several steps which we formulate as lemmas.

Lemma 1. Let the assumptions of Theorem $B$ be valid. Then the operator T defines a nuclear operator $T_{\mathbb{R}}$ of order zero in the real Banach space $A_{\infty}\left(\Omega_{\mathbb{R}}\right)$ whose trace is given by trace $T_{\mathbb{R}}=\sum_{k=1}^{m} \varphi_{k}\left(z_{k}^{*}\right) \operatorname{det}\left(1-\psi_{k}^{\prime}\left(z_{k}^{*}\right)\right)^{-1}$.

Proof. The proof follows immediately from the proofs of the same statements about the operator $T$ in the space $A_{\infty}(\Omega)$ [5]. We have shown that $T$ can be represented in $A_{\infty}(\Omega)$ as

$$
T=\sum_{\{i\}} \varrho_{i} f_{i}^{*} \otimes f_{i}
$$

$\sum_{\{i\}}\left|\varrho_{i}\right|^{\alpha}<\infty$ for all $\alpha>0$ and $f_{i}^{*} \in A_{\infty}(\Omega)^{*}, f_{i} \in A_{\infty}(\Omega)$ can be chosen such that $\left\|f_{i}^{*}\right\|=\left\|f_{i}\right\|=1$. Now under the assumptions of Theorem $\mathrm{B}$ it is fairly easy to see that in fact the $\varrho_{i}$ can be chosen real, the $f_{i} \in A_{\infty}\left(\Omega_{\mathbb{R}}\right)$ and the $f_{i}^{*}$ real valued on $A_{\infty}\left(\Omega_{\mathbb{R}}\right)$. Therefore we have for the operator $T_{\mathbb{R}}$ exactly the same representation. This shows immediately that the trace of $T_{\mathbb{R}}$ is equal to the trace of $T$ which was shown to be just the expression given in the lemma. 
We want to show next that the spectra of the operators $T$ and $T_{\mathbb{R}}$ are the same. First remember, that the spectrum of the operator $T_{\mathbb{R}}$ in the real space $A_{\infty}\left(\Omega_{\mathbb{R}}\right)$ is by definition the spectrum of the operator $T_{\mathbb{R}}$ in the complexification $A_{\infty}\left(\Omega_{\mathbb{R}}\right)$ $+i A_{\infty}\left(\Omega_{\mathbb{R}}\right)$ of the space $A_{\infty}\left(\Omega_{\mathbb{R}}\right)$. It is clear that $T_{\mathbb{R}}$ is also a nuclear operator of order zero in this complex space which is trivially a subspace of the Banach space $A_{\infty}(\Omega)$.

So every eigenvalue of the operator $T_{\mathbb{R}}$ is also an eigenvalue of the operator $T$ in $A_{\infty}(\Omega)$.

Denote by $\Lambda$ the set of eigenvalues of $T$ different from zero which are not contained in the spectrum $\sigma\left(T_{\mathbb{R}}\right)$ of $T_{\mathbb{R}}$. Because both operators are nuclear of order zero we have

$$
\operatorname{trace} T_{\mathbb{R}}^{n}=\sum_{\lambda \in \sigma\left(T_{\mathbb{R}}\right)} \lambda^{n} \text { and } \operatorname{trace} T^{n}=\sum_{\lambda \in \sigma\left(T_{\mathbb{R}}\right)} \lambda^{n}+\sum_{\lambda \in A} \lambda^{n} .
$$

Because of Lemma 1 we then get for every $n \geqq 1$

$$
\sum_{\lambda \in \Lambda} \lambda^{n}=0
$$

We show next

Lemma 2. Let $\Lambda$ be a set of complex numbers such that $\sum_{\lambda \in \Lambda}|\lambda|<\infty$ and $\sum_{\lambda \in \Lambda} \lambda^{n}=0$ for all $n \geqq 1$. Then $\lambda=0$ for all $\lambda \in \Lambda$.

Proof. Because $\sum_{\lambda \in A}|\lambda|<\infty$ we know that for $z$ small enough the following function $g$ is holomorphic:

$$
g(z):=\exp \sum_{n=1}^{\infty} \frac{1}{n} \sum_{\lambda \in \Lambda} \lambda^{n} z^{n} .
$$

Because $\sum_{\lambda \in A} \lambda^{n}=0$ for all $n$ we have trivially $g(z) \equiv 1$. On the other hand we can calculate for small enough $z$ the right hand side and get

$$
\exp \sum_{n=1}^{\infty} \frac{1}{n} \sum_{\lambda \in A} \lambda^{n} z^{n}=\prod_{\lambda \in A}(1-\lambda z)^{-1} .
$$

Because the left hand side is an entire function in the whole $z$-plane also the right hand side must be entire. But this is possible only for $\lambda=0$ for all $\lambda \in A$. This shows that the following lemma is true.

Lemma 3. The spectra of the operators $T$ and $T_{\mathbb{R}}$ are the same.

The discussion of the spectrum of the operator $T$ is therefore reduced to that of the operator $T_{\mathbb{R}}$ which we will do next.

Lemma 4. The operator $T_{\mathbb{R}}: A_{\infty}\left(\Omega_{\mathbb{R}}\right) \rightarrow A_{\infty}\left(\Omega_{\mathbb{R}}\right)$ is $u_{0}$-positive.

Proof. We first define a cone $K$ in the space $A_{\infty}\left(\Omega_{\mathbb{R}}\right)$ :

$$
K:=\left\{f \in A_{\infty}\left(\Omega_{\mathbb{R}}\right): f(z) \geqq 0 \text { for } z \in \Omega_{\mathbb{R}}\right\} .
$$


It is clear that $K$ is a proper reproducing cone in the space $A_{\infty}\left(\Omega_{\mathbb{R}}\right)$. Because $\varphi_{k}(z)>0$ for $z \in \Omega_{\mathbb{R}}$ and $\overline{\psi_{k}\left(\Omega_{\mathbb{R}}\right)} \subset \Omega_{\mathbb{R}}$ for all $k=1, \ldots, m$ it follows also that $T_{\mathbb{R}}$ leaves the cone $K$ invariant and is therefore positive. Let be $u_{0}=1$ the holomorphic function constant one. Then clearly $u_{0} \in K$ and $u_{0} \neq 0$. Let be $f \in K$ and $f \neq 0$.

Denote further by $M_{f}:=\sup _{z \in \bar{\Omega}}|T f(z)|$. Then we have

$$
T_{\mathbb{R}} f \leqq M_{f} u_{0},
$$

which shows that $T_{\mathbb{R}}$ is $u_{0}$-upper bounded. We want to show that $T_{\mathbb{R}}$ is also $u_{0}$-lower bounded. For this we use the fact that for any holomorphic function $f$ of $n$ complex variables $z=\left(z_{1}, \ldots, z_{n}\right)$ the set $N(f):=\{z \in \Omega: f(z)=0\}$ consists in every region within its domain of holomorphy of a finite number $r$ of $(2 n-2)$ dimensional analytic sets $G_{1}, \ldots, G_{r}$ which do not have an accumulation point within this bounded region [17]. Therefore also in every region $\tilde{H}$ strictly contained in $\Omega_{\mathbb{R}}$ there can be only a finite number $r$ of analytic sets $\tilde{G}_{i}:=G_{i} \cap \Omega_{\mathbb{R}}$, which are the intersection of the analytic sets $G_{1}, \ldots, G_{r}$ of $f$ in a set $H$ in $\Omega$ such that $\tilde{H}=H \cap \Omega_{\mathbb{R}}$.

From the properties of the functions $\psi_{k}$ it follows then that the function $T_{\mathbb{R}} f(z)$ has in the region $\bar{\Omega}$ a finite number $r$ of analytic sets $G_{i}, i=1 \ldots r$ as its nullsets. [Assuming that the functions $\varphi_{k}(z)$ can also be analytically continued to a certain neighbourhood $\Omega_{1}$ containing strictly $\bar{\Omega}$.] Therefore also in $\bar{\Omega}_{\mathbb{R}}$ there are a finite number $r(f)$ of analytic sets $\widetilde{G}_{i}=G_{i} \cap \bar{\Omega}_{\mathbb{R}}$ on which $T_{\mathbb{R}} f(z)$ vanishes. We claim that under the assumptions of the Theorem B there exists a $p \in \mathbb{N}$ such that $T_{\mathbb{R}}^{p} f$ has no zero at all in $\bar{\Omega}_{\mathbb{R}}$. Let us assume that this is not the case. Then there exists to every $N \in \mathbb{N}$ an analytic set $G$ in $\bar{\Omega}$ such that $\tilde{G}=G \cap \bar{\Omega}_{\mathbb{R}}$ is not empty and $T_{\mathbb{R}}^{N} f(z)=0$ for $z \in \tilde{G}$. Let us write explicitly the expression for $T_{\mathbb{R}}^{N} f(z)$ which looks like this

$$
T_{\mathbb{R}}^{N} f(z)=\sum_{\substack{\boldsymbol{\alpha}_{1}, \ldots, \boldsymbol{\alpha}_{m} \\\left|\boldsymbol{\alpha}_{1}\right|+\ldots+\left|\boldsymbol{\alpha}_{m}\right|=N-1}} \varphi_{\boldsymbol{\alpha}_{1}, \ldots, \boldsymbol{\alpha}_{m}}(z)(T f)\left(\psi^{\boldsymbol{\alpha}_{1}, \ldots, \boldsymbol{\alpha}_{m}}(z)\right) .
$$

The functions $\varphi_{\boldsymbol{\alpha}_{1}, \ldots, \boldsymbol{\alpha}_{m}}(z)$ are certain products of the functions $\varphi_{k}, k=1, \ldots, m$ with arguments $\psi^{\boldsymbol{\beta}_{1}, \ldots, \boldsymbol{\beta}_{m}}(z)$, where $\boldsymbol{\beta}_{1}, \ldots, \boldsymbol{\beta}_{m}$ depend on $\boldsymbol{\alpha}_{1}, \ldots, \boldsymbol{\alpha}_{m}$ in a certain way the explicite form of which we are not interested. We only need that the functions $\varphi_{\boldsymbol{\alpha}_{1}, \ldots, \boldsymbol{\alpha}_{m}}$ are all strictly positive on $\bar{\Omega}_{\mathbb{R}}$. Therefore on $\Omega_{\mathbb{R}}$ we find

$$
T_{\mathbb{R}}^{N} f(z)=0 \nsim\left(T_{\mathbb{R}} f\right)\left(\psi^{\alpha_{1}, \ldots, \alpha_{m}}(z)\right)=0,
$$

where the mappings $\psi^{\alpha_{1}, \ldots, \alpha_{m}}$ had been defined in (8). Therefore $T_{\mathbb{R}} f$ vanishes on all $\psi^{\alpha_{1}, \ldots, \alpha_{m}}(\tilde{G})$. Because the $\psi_{k}, k=1, \ldots, m$, have been chosen as separating we can find for $z \in \tilde{G}$ a $p>0$ with $\psi^{\boldsymbol{\beta}_{1}, \ldots, \boldsymbol{\beta}_{m}}(z) \notin \tilde{G}_{i}$ for all $1 \leqq i \leqq r$ with some $\boldsymbol{\beta}_{1}, \ldots, \boldsymbol{\beta}_{m}$ with $\left|\boldsymbol{\beta}_{1}\right|+\ldots+\left|\boldsymbol{\beta}_{m}\right|=p$. But this is in contradiction to our assumption that the function $T_{\mathbb{R}} f(z)$ vanishes in $\bar{\Omega}_{\mathbb{R}}$ just only on $\tilde{G}_{i}, i=1, \ldots, r(f)$. Therefore there must exist a $q \in \mathbb{N}$ such that $T_{\mathbb{R}}^{q} f(z)$ does not vanish on $\bar{\Omega}_{\mathbb{R}}$. Let be $\beta:=\min _{z \in \bar{\Omega}_{\mathbb{R}}} T_{\mathbb{R}}^{q} f(z)$. Because of the above discussion we know $\beta>0$. Therefore we get

$$
T_{\mathbb{R}}^{q} f \geqq \beta u_{0}
$$


This together with (13) shows that $T_{\mathbb{R}}$ is $u_{0}$-positive in $A_{\infty}\left(\Omega_{\mathbb{R}}\right)$. Especially for $f=u_{0}$ we get

$$
T_{\mathbb{R}} u_{0} \geqq \min _{z \in \bar{\Omega}_{\mathbb{R}}} \sum_{k=1}^{m} \varphi_{k}(z) .
$$

Combining Lemmas 1, 3, and 4 we have proven Theorem B.

\section{An Example from Statistical Mechanics}

Consider a one-dimensional spin lattice system where the spin variable $\sigma$ can take on a discrete set $\sigma_{i}, i \in I$ of values. Assume that the interaction $V$ of two spins $\sigma_{i}, \sigma_{j}$ at lattice sites separated a distance $l \in \mathbb{N}$ is given by

$$
V\left(\sigma_{i}, \sigma_{j}\right)=\sum_{r=1}^{n} c_{r} \lambda_{r}^{l} \sigma_{i} \sigma_{j}, \quad 0<\lambda_{r}<1
$$

and $\lambda_{k} \neq \lambda_{s}$ for $s \neq k, c_{r} \in \mathbb{R}$.

Then the transfer matrix $L$ of this system has the following form [2]

$$
L f\left(z_{1}, \ldots, z_{n}\right)=\sum_{j \in I} f\left(\left\{\lambda_{k} \sigma_{j}+\lambda_{k} z_{k}\right\}\right) \exp -\sigma_{j} \sum_{r=1}^{n} \mathrm{c}_{r} z_{r} .
$$

If one choses the open set $\Omega \subset \mathbb{C}^{n}$ in an appropriate way [2] this $L$ defines a nuclear operator in $A_{\infty}(\Omega)$.

If we set $\varphi_{k}(z):=\exp -\left(\sigma_{k} \sum_{r=1}^{n} c_{r} z_{r}\right)$ and $\psi_{k}(z)=\left(\left\{\lambda_{r} \sigma_{k}+\lambda_{r} z_{r}\right\}\right)$ for $k \in I$ we see that the assumptions of Theorem B are fulfilled. (We use $\lambda_{i} \neq \lambda_{j}$ for at least one pair of indices $i, j \in I$ to show that the $\psi_{k}$ are separating.)

Our Theorem B shows therefore that the highest eigenvalue $\lambda_{0}$ of $L$ is simple and in absolute value bigger than all other eigenvalues. Therefore $\lambda_{0}$ is in fact analytic in the constants $c_{r}$ which are the interesting physical parameters.

Let us finally add some comments. It is clear that one can handle in exactly the same way as above also operators of the form

$$
T f(z)=\sum_{k=1}^{\infty} \varphi_{k}(z) f \circ \psi_{k}(z)
$$

as long as this defines a reasonable compact operator. Another interesting case arises if $z$ is not any more in a finite dimensional complex space but varies instead in some open bounded domain of some complex Banach space B. As we said in the introduction also this case can be treated by the method explained above. One only has to replace the statements about functions holomorphic in finitely many variables by the analogous facts for holomorphic functions in Banach spaces, as long as they are known. Otherwise one has to add to the formulation of Theorem B the relevant assumptions which enable us to carry out the proof in a similar way [5]. (We have in mind there especially the problem of sets of uniqueness in Banach spaces.) 
That the property of the $\psi_{k}$ 's of being separating is indeed necessary for Theorem B to hold can be seen from the following example: Take the operator $L$ in (18) with $n=2$ and $c_{r}=0$ for all $r$. Let $\lambda_{1}=\lambda_{2}=\lambda$. Then the mappings $\psi_{k}$ are not any more separating as can easily be seen. The operator $L$ for this case looks like follows:

$$
(L f)\left(z_{1}, z_{2}\right)=\sum_{j \in I} f\left(\lambda \sigma_{j}+\lambda z_{1}, \lambda \sigma_{j}+\lambda z_{2}\right) .
$$

There exist infinitely many eigenfunctions in the cone $K$ which are given by the functions $f_{n}\left(z_{1}, z_{2}\right)=\left(z_{1}-z_{2}\right)^{2 n}$. The corresponding eigenvalues are just $\lambda_{n}=|I| \lambda^{2 n}$.

So the unicity of the positive eigenvector is lost. What presumably is still true is the fact that the highest eigenvalue is still simple. But this we can not show by our method.

\section{References}

1. Viswanathan, K.S.: Statistical mechanics of a one-dimensional lattice gas with exponential polynomial interactions. Commun. Math. Phys. 47, 131-141 (1976)

2. Mayer, D.H.: The transfer-matrix of a one-sided subshift of finite type with exponential interaction. Lett. Math. Phys. 1, 335-343 (1976)

3. Mayer, D.H., Viswanathan, K.S. : On the $\zeta$-function of a one-dimensional classical system of hardrods. Commun. Math. Phys. 52, 175-189 (1977)

4. Mayer, D.H., Viswanathan, K.S. : Statistical mechanics of one-dimensional Ising and Potts models with exponential interactions. Physica 89 A, 97-112 (1977)

5. Mayer, D.H.: On composition operators on Banach spaces of holomorphic functions. Preprint, RWTH Aachen (1978); J. Funct. Anal. (in press)

6. Kac, M.: Mathematical mechanisms of phase transitions. In: Brandeis University Summer Institute in Theoretical Physics, Vol. 1, pp. 243-305. London: Gordon and Breach 1966

7. Perron, O.: Zur Theorie der Matrizen. Math. Ann. 64, 248-263 (1907) Frobenius, G.: Über Matrizen aus nicht negativen Elementen. S.-B. Preuss. Akad. Wiss. Berlin 456-477 (1912)

8. Jentzsch, R.: Über Integraloperatoren mit positivem Kern. Crelles J. Math. 141, 235-244 (1912)

9. Krein, M.G., Rutman, M.A.: Linear operators leaving invariant a cone in a Banach space. Transl. Am. Math. Soc. Ser. 1, 10, 199-325 (1950)

10. Krasnoselskii, M.A., Ladyzenskii, L.A.: Structure of a spectrum of positive non-homogeneous operators. Tr. Mosk. Mat. Obscestva 3, (1954)

11. Krasnoselskii, M.A.: Positive solutions of operator equations, Chap. 2. Groningen, The Netherlands: Noordhoff 1964

12. Schäfer, H.H.: Topological vector spaces. Berlin, Heidelberg, New York: Springer 1971

13. See [11], p. 62, Theorem 2.2

14. See [11], Theorems $2.5,2.10,2.11$, and 2.13

15. Dieudonne, J.: Foundations of modern analysis, p. 209. New York: Academic Press 1969

16. Herve, M.: Several complex variables, local theory, p. 83. Oxford: Oxford University Press 1963

17. Behnke, H., Thullen, P.: Theorie der Funktionen mehrerer komplexer Veränderlichen, Chap. V, 2. Aufl. Berlin, Heidelberg, New York: Springer 1970

Communicated by H. Araki

Received October 24, 1978; in revised form February 17, 1979 